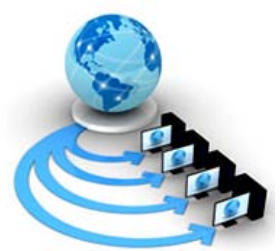

Volume 8, No. 7, July - August 2017

International Journal of Advanced Research in Computer Science

RESEARCH PAPER

\title{
HANDWRITTEN DEVANAGARI VOWEL RECOGNITION USING ARTIFICIAL NEURAL NETWORK
}

\author{
P E Ajmire \\ Head \& Associate Professor \\ Department of Computer Science \& Application \\ G. S. Science, Arts \& Commerce College, Khamgaon. 444303
}

\begin{abstract}
Handwritten character recognition is active and open field of research in the area of pattern recognition. As, there is continuous developments in the hardware i.e. Machine. At the same time, many researchers are working in the field of character recognition from more than last four decays. Handwritten character recognition involves reading of handwritten character and comparing the required one. Human being is doing this task while learning characters in the childhood. But the same task for machine is much complex. This complexity depends on the environment in which the character was written. For the machine the process of reading, understanding and interpretation of handwritten character is difficult task. This research work proposes new approaches for extracting features in context of Handwritten Devanagari Vowels recognition. For classification technique Artificial Network is used. The overall accuracy of recognition of handwritten Devanagari Vowels is $\%$ with SVM classifier, \% with MLP and it is \%with GFF.
\end{abstract}

Keyword: Handwritten, Devanagari Vowel, Feature Extraction, Artificial Neural Network.

\section{INTRODUCTION}

At present many researchers are working on handwritten Devanagari characters. Devanagari script is the most popular script. It is phonetic in nature and the writing system for this script maps the sound of the 'Aksharas' to specific shapes. Devanagari is used in many Indian languages like Sanskrit, Hindi, Nepali, Marathi, Konkani, Sindhi etc. More than 300 million people around the world use Devanagari script [1]. From last four decays many researchers are working for the development of system of fully automating the process of reading, understanding and interpretation of handwritten documents. Huge and important historical document is available in the libraries and museums in the world. The preservation this heritage is necessary. For the preservation of such document, it should be digitalized. So, this will be available worldwide through large on line digital libraries. Historical documents are of more importance because they are a significant part of our cultural heritage. During, the last decades a lot of research has been done in the field of Optical Character Recognition (OCR). The available OCR text recognition technologies are unable to meet the accuracy in recognition. Documents are in the form of papers which human can read and understand easily but for the machine it is difficult to read and understand these documents directly. It is demand of time to develop a system, which includes understanding and interpretation of handwritten character. There are thousands of languages throughout the world and character recognition complexity differs from language to language. Devanagari character recognition is a complicated task due to the presence of multiple loops, conjuncts, modifiers (upper and lower), character variations (disconnected and multi-stroke) and also because some writers use header line. Similarly the way of writing also depends on the environment and the mood of writer. There is a wide verity of size and shape of each character written by writer. As handwriting is free style of writing, it is unconstrained. Devanagari there are vowels, consonants, vowel modifiers and component characters, numerals, etc, moreover, there are many similar shaped characters. All these variations make the handwritten character recognition, a challenging task [2, 3].

\section{REVIEW OF LITERATURE}

The research work on character recognition of Devanagari script had been started in 1970, where Sinha and Mahabala [4] presented a syntactic pattern analysis system for the recognition of Devanagari Characters (DC). First research report on handwritten Devanagari Characters (HDC) had published in 1977 by Sethi and Chatterjee [5], however, very few research papers published on OCR during that period. The computer processing power enhanced rapidly after 1990 and cost decreases drastically, which boost the research in image processing field. During this period (1995 on word) an extensive research work on printed Devanagari Characters and Handwritten Characters was carried out by Bansal et.al [6]. Mrs. A P Jane et al. [7] propose a novel method to recognize handwritten Marathi characters of similar shaped using Artificial Neural Network and recorded average accuracy $70 \%$ to $90 \%$. Akhil Deshmukh et al[8] proposed a new approach of Eigen space method which uses the concept of Gerschgorin's theorems in order to recognize and extract the characters. Mahesh Jangid et al [9] proposed new feature extraction algorithm Correlation of Gradients in Local Neighbors (CGLN) for the handwritten character recognition. Using these feature method and SVM classifier, they obtained $95.38 \%$ accuracy for handwritten Devanagari character recognition. 


\section{FEATURE EXTRACTION}

The handwritten characters have large variation in shapes within a class of character. This variation depends from font styles, document noise, photometric effect, document skew and poor image quality. The large variation in shapes makes it difficult to determine the number of features that are convenient prior to model building. The performance of a character recognition system depends heavily on what features are being used [10]. Hence, the feature extraction method is the important task. The task begins with the preparation of database. This handwritten database is developed with the help of students, person and housewives of different educational background and of different mother tongues. In offline handwritten numeral recognition, the handwritten document has to be scan and store as image for processing. The datasheet paper is scanned and after the preprocessing [11] the scanned images are stored in database. Every vowel is converted into image form. The sample database of 13 vowels is shown in figure 1.
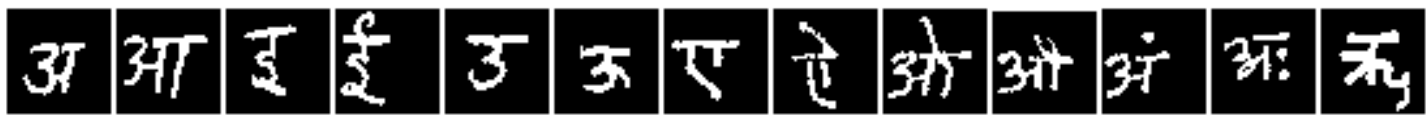

Fig.1: Sample database of Devanagari Vowels.

For this research work histogram oriented gradient features are extracted. The extracted features are selected for the classification. HOG features describe the shape of the image by the distribution of intensity gradients or edge directions. A HOG feature vector represents local shape of an object $[12,13]$. HOG features are relatively invariant to local geometric and photometric transformations.

\section{CLASSIFICATION}

Support vector machine is a supervised classification; basically it is a statistical classification. It is well known as binary classifier [14]; it can be adopted for multiple class tasks. The support vectors machine is effective on high dimensional data as it supports vectors lie closest to the decision boundary. SVM with a small number of support vectors can have good generalization, even when the dimensionality of the data is high [15]. SVM is also applicable to high dimensional space under small training sample conditions. SVMs have been successfully applied to a number of applications ranging from face detection, verification, and recognition. Multilayer perceptron (MLPs) are layered feed forward networks typically trained with static back propagation. MLP has been chosen because of its well-known learning and generalization abilities, which is necessary for dealing with imprecision in input patterns [16]. Architecturally, an MLP is a feed-forward layered network of artificial neurons. Each artificial neuron in the MLP computes a TanhAxon and Sigmoid function of the weighted sum of all its inputs. An MLP consists of one input layer, one output layer and one hidden or every neuron in a layer of the MLP is connected to all inputs of each neuron in the immediate next layer of the same. These networks have found their way into countless applications requiring static pattern classification. Principal component analysis networks (PCAs) combine unsupervised and supervised learning in the same topology. Principal component analysis is an unsupervised linear procedure that finds a set of uncorrelated features, principal components, from the input. Generalized feed forward (GFF) networks are a generalization of the MLP such that connections can jump over one or more layers. In theory, a MLP can solve any problem that a generalized feed forward network can solve. In practice, however, generalized feed forward networks often solve the problem much more efficiently.

\section{RESULT AND DISCUSSION}

All the text images are labeled for feature extraction and classification. The table 1 shows the name of images used for this work.

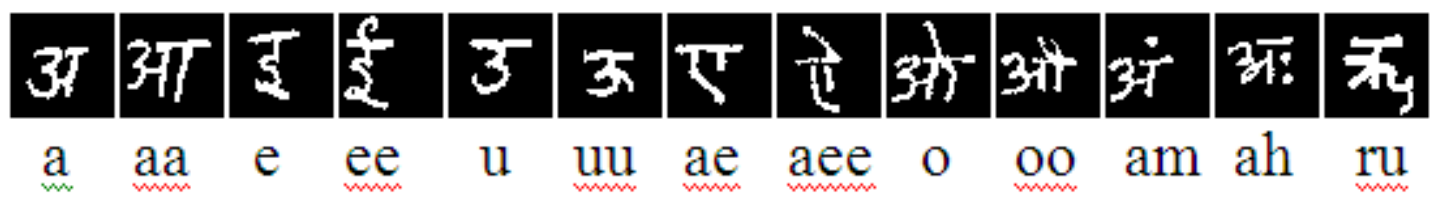

Table 1: Labeled database of Devanagari Vowels.

From the data set, $60 \%$ data is reserved for training, $15 \%$ for cross validation and $25 \%$ for testing. The obtained results of classification of 13 vowels using SVM classifier are shown in following table 2.

\begin{tabular}{|c|c|c|c|c|c|c|c|c|c|c|c|c|}
\hline & \multicolumn{12}{|c|}{ Classifiers } \\
\hline & \multicolumn{3}{|c|}{ MLP } & \multicolumn{3}{|c|}{ PCA } & \multicolumn{3}{|c|}{ GFF } & \multicolumn{3}{|c|}{ SVM } \\
\hline Vowel & Test & $\mathrm{CV}$ & Training & Test & $\mathrm{CV}$ & Training & Test & $\mathrm{CV}$ & Training & Test & $\mathrm{CV}$ & Training \\
\hline (e) & 50.00 & 77.78 & 100.00 & 62.50 & 77.78 & 92.31 & 50.00 & 77.78 & 100.00 & 62.50 & 88.89 & 100.00 \\
\hline (ae) & 62.50 & 71.43 & 100.00 & 50.00 & 85.71 & 100.00 & 75.00 & 71.43 & 100.00 & 87.50 & 100.0 & 100.00 \\
\hline (аеe) & 100.0 & 100.0 & 100.00 & 87.50 & 100.0 & 94.44 & 100.0 & 100.0 & 100.00 & 100.0 & 100.0 & 100.00 \\
\hline (am) & 66.67 & 100.0 & 90.91 & 100.0 & 100.0 & 100.00 & 100.0 & 100.0 & 100.00 & 100.0 & 100.0 & 100.00 \\
\hline
\end{tabular}




\begin{tabular}{|c|c|c|c|c|c|c|c|c|c|c|c|c|} 
(ah) & 44.44 & 100.0 & 94.12 & 77.78 & 100.0 & 88.24 & 66.67 & 75.00 & 100.00 & 77.78 & 75.00 & 100.00 \\
\hline (aa) & 90.00 & 100.0 & 100.00 & 90.00 & 100.0 & 100.00 & 90.00 & 100.0 & 100.00 & 100.0 & 75.00 & 100.00 \\
\hline (o) & 80.00 & 83.33 & 100.00 & 60.00 & 66.67 & 94.74 & 80.00 & 83.33 & 100.00 & 80.00 & 83.33 & 100.00 \\
\hline (ee) & 45.45 & 100.0 & 100.00 & 54.55 & 50.00 & 100.00 & 72.73 & 75.00 & 100.00 & 63.64 & 100.0 & 100.00 \\
\hline (a) & 40.00 & 0.00 & 86.36 & 40.00 & 33.33 & 81.82 & 60.00 & 66.67 & 95.45 & 60.00 & 66.67 & 100.00 \\
\hline (ru) & 100.0 & 100.0 & 100.00 & 100.0 & 100.0 & 100.00 & 100.0 & 100.0 & 100.00 & 100.0 & 100.0 & 100.00 \\
\hline (u) & 80.00 & 100.0 & 100.00 & 100.0 & 100.0 & 100.00 & 80.00 & 100.0 & 100.00 & 100.0 & 66.67 & 100.00 \\
\hline (uu) & 83.33 & 100.0 & 100.00 & 83.33 & 100.0 & 95.24 & 66.67 & 100.0 & 100.00 & 83.33 & 100.0 & 100.00 \\
\hline $\mathbf{( o o})$ & 87.50 & 83.33 & 100.00 & 100.0 & 83.33 & 93.75 & 75.00 & 83.33 & 100.00 & 100.00 & 83.33 & 100.00 \\
\hline Avg\% & $\mathbf{7 1 . 5 3}$ & $\mathbf{8 5 . 8 4}$ & $\mathbf{9 7 . 8 0}$ & $\mathbf{7 7 . 3 6}$ & $\mathbf{8 4 . 3 7}$ & $\mathbf{9 5 . 4 3}$ & $\mathbf{7 8 . 1 6}$ & $\mathbf{8 7 . 1 2}$ & $\mathbf{9 9 . 6 5}$ & $\mathbf{8 5 . 7 4}$ & $\mathbf{8 7 . 6 0}$ & $\mathbf{1 0 0 . 0 0}$ \\
\hline
\end{tabular}

Table 2: Obtained Results for various Classifiers.

From the above obtained result, it is observed that the handwritten Vowel recognition using SVM classifier on training data is $100 \%$, where as it is $85.74 \%$ on testing data and $87.60 \%$ on cross validation. The average recognition accuracy of all handwritten Devanagari vowels for MLP, PCA, GFF and SVM on Testing, CV and Training data are shown in following figure 2.

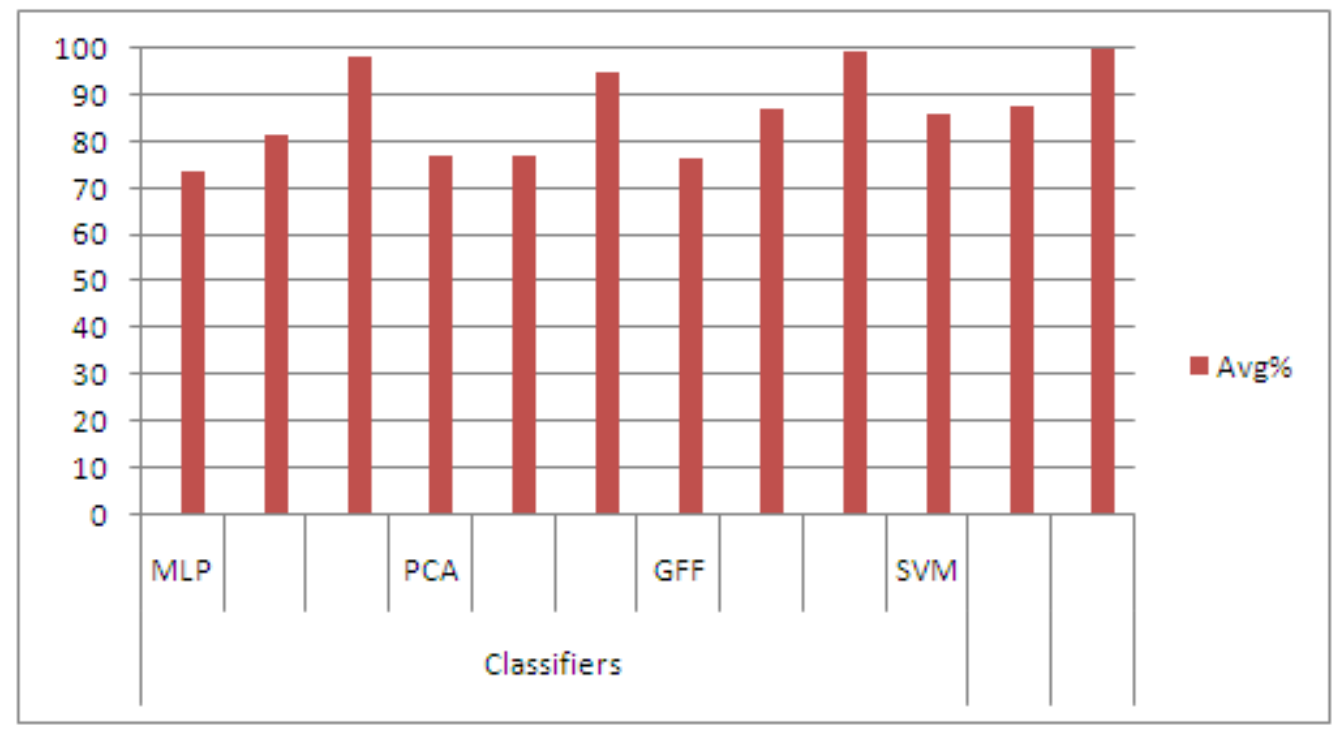

Fig 2: Average recognition of all Vowels for various classifier.

\section{REFERENCES}

1]. Latesh Malik and P. S. Deshpande, "Recognition of printed Devnagari characters with regular expression in finite state models", Proceedings of the International Workshop on Machine Intelligence Research (MIR Day, GHRCE- Nagpur), 2009.

2]. Mahesh Jangid, "Devanagari Isolated Character Recognition by using Statistical features", International Journal on Computer Science and Engineering (IJCSE), Vol. 3(6), June 2011.

3]. D H Lentz and R Sowerby, "Feature extraction of concave and convex regions and their intersections", Computer Aided Design, published by Elsevier, Vol.25(7), pp. 421-437, July 1993.

4]. Sinha R.K., Mahabala, "Machine Recognition of Devanagari Script”, IEEE Trans. System Man Cyber, pp.435-441, 1979.

5]. I.K. Sethi, B. Chatterjee, "Machine recognition of constrained hand printed Devanagari”, Pattern Recognition, The Journal of the Pattern Recognition Society, Vol. 9(2), pp. 69-75, July 1977.

6]. V. Bansal, "Integrating Knowledge Sources in Devanagari Text Recognition. IIT, Kharagpur, Ph.D. Thesis, 1999.

7]. Mrs. A P Jane and M A Pund, "Recognition of Similar Shaped Handwritten Marathi characters using Artificial
Neural Network”, Global Journal of Computer Science and Technology Vol. XII, Issue XI, 2012.

8]. Akhil Deshmukh, Rahul Meshram, Sachin Kendre, Kunal Shah, "Handwritten Devanagari Character Recognition", International Journal of Engineering Research \& Technology, Vol. 3 Issue 4, April - 2014.

9]. Mahesh Jangid, Sumit Srivastava, "Handwritten Devanagari characters recognition using correlation of gradients in local neighbors”, Journal of Scientific Research and Development Vol.3 (1),pp: 107-114, 2016.

10]. P. E. Ajmire, R. V. Dharaskar, V. M. Thakare and S E Warkhede, "Structural Features for Character Recognition System-A Review", International Journal of Advanced Research in Computer Science (IJARCS), Volume 3, No. 3, June 2012.

11]. N Dalal and B Triggs, “ Histogram oriented gradient for human detection”, IEEE Computer Society Conference on Computer Vision and Pattern Recognition, 2005.

12]. Takuya Kobayashi, Akinori Hidaka, and Takio Kurita, "Selection of Histograms of Oriented Gradients Features for Pedestrian Detection”, International Conference on Neural Information Processing (ICONIP), LNCS Vol. 4985, pp. 598607, 2008.

13]. Parshuram M. Kamble , Ravinda S. Hegadi, "Handwritten Marathi character recognition using R-HOG Feature", 
International Conference on Advanced Computing Technologies and Applications, Elsevier, Procedia Computer Science 45 pp.266 - 274, 2015

14]. Chih-Wei Hsu and Chih-Jen Lin, "A Comparison of Methods for Multi-class Support Vector Machines”, Department of Computer Science and Information Engineering National Taiwan University Taipei 106, Taiwan.

15]. Koby Crammer and Yoram Singer, "On the Algorithmic Implementation of Multiclass Kernel-based Vector
Machines”, Journal of Machine Learning Research 2, pp.265292, 2001.

16]. Sandhya Arora, D. Bhattacharjee, M. Nasipuri, D. K. Basu and M. Kundra, "Complementary Features Combined in a MLP-based System to Recognize Handwritten Devanagari Characters" Journal of Information Hiding and Multimedia Signal Processing Vo. 2,No.1 Jan 2011. 